\title{
Risk Factors for Delayed Hyperthyrotropinemia in Late Preterm Infants
}

Mun Hui Jeong, $\mathrm{MD}^{1}$, Narae Lee, $\mathrm{MD}^{1}$, Mi Hye Bae, $\mathrm{MD}^{2}$, Young Mi Han, $\mathrm{PhD}^{1}$, Kyung Hee Park, $\mathrm{PhD}^{2}$, and Shin Yun Byun, $\mathrm{PhD}^{1}$

${ }^{1}$ Department of Pediatrics, Pusan National University Children's Hospital, Pusan National University School of Medicine, Yangsan, Korea

${ }^{2}$ Department of Pediatrics, Pusan National University Hospital, Pusan National University School of Medicine, Busan, Korea

\section{ABSTRACT}

Purpose: This study aimed to investigate the prevalence of delayed thyroid dysfunction based on iodine disinfectant use and to analyze associated risk factors.

Methods: A retrospective study was conducted on late preterm infants admitted to the neonatal intensive care unit between January 2010 and June 2018, who underwent neonatal thyroid screening (NTS) and $\geq 2$ thyroid function tests (TFTs). NTS was performed 3 days after birth, with at least two TFTs 1 week and 2 to 4 weeks after birth. To distinguish between normal and dysfunctional thyroid levels, we reviewed TFT results at 2 to 4 weeks and examined possible risk factors for the development of thyroid dysfunction.

Results: Of 295 late preterm infants, 262 were enrolled with a mean gestational age and birth weight of $34.8 \pm 0.7$ weeks and $2,170 \pm 454 \mathrm{~g}$, respectively. A total of $7.6 \%$ developed hyperthyrotropinemia at the age of $24.3 \pm 14.6$ days (range, 12 to 69 ). The incidence of hyperthyrotropinemia during iodine use was approximately $12.6 \%$, while that during discontinuation was $2.4 \%(P=0.002)$. Multivariate analysis revealed that small for gestational age (SGA), iodine disinfectant use, and abnormal NTS results were significant risk factors for delayed hyperthyrotropinemia (adjusted odds ratio [AOR]: 4.27, $P=0.008$; AOR: $8.24, P=0.003$; and AOR: $7.80, P=0.002$, respectively).

Conclusion: Delayed hyperthyrotropinemia was prevalent in late preterm infants exposed to topical iodine and those identified as being SGA. Secondary TFTs should be considered 2 to 4 weeks after birth for this population at risk.

Key Words: Congenital hypothyroidism; Iodine; Infant, premature; Small for gestational age; Thyrotropin

\section{INTRODUCTION}

Late preterm infants (born at 34 to 36 weeks gestation) account for approximately $80 \%$ of preterm infants worldwide and are expected to occupy a relatively high percentage of the
Received: 9 July 2019

Revised: 24 August 2019

Accepted: 19 September 2019

Correspondence to: Young Mi Han, PhD

Department of Pediatrics, Pusan National University Children's Hospital, Pusan National University School of Medicine, 20 Geumo-ro, Mulgeumeup, Yangsan 50612, Korea

Tel: +82-55-360-2180

Fax: $+82-55-360-2181$

E-mail: skybluehym@gmail.com

Copyright(c)

By Korean Society of Neonatology.

All right reserved.

This is an Open-Access article distributed under the terms of the Creative Commons Attribution Non-Commercial License (http://creativecommons.org/licenses/ by-nc/4.0), which permits unrestricted non-commercial use, distribution, and reproduction in any medium, provided the original work is properly cited. 
population in the coming years ${ }^{1)}$. This group had been called "near term" but was renamed as "late preterm," recognizing the importance of the development in the last 6 weeks of pregnancy ${ }^{2-4)}$. Over the past 10 years, some studies have observed delayed development and cognitive decline in late preterm infants ${ }^{5-7)}$. However, it is difficult to determine the factors that contribute to this delayed development since hospitalization criteria for these patients differ across units ${ }^{8,9)}$ and these infants are often not followed. A study conducted by Cuestas et al. ${ }^{10)}$ showed that these infants are significantly disadvantaged due to the long-term effects of hyperthyrotropinemia that persist until childhood.

Thyroid hormones play a diverse role in the maintenance of homeostasis and are critical for neurodevelopment, particularly during the fetal and neonatal periods ${ }^{11}$. Hypothyroidism during this period can cause unrecognized cognitive disorders; however, with the introduction of neonatal thyroid screening (NTS) policies, these irreversible cognitive impairments can now be significantly minimized to prevent further medical complications ${ }^{12)}$. However, data gathered over the past two decades suggest that due to delayed thyroid dysfunction in premature infants, one NTS program is insufficient to estimate the effects on future de velopment. Delayed thyroid dysfunction is known to develop due to hypothalamus-pituitary-thyroid axis immaturity, various diseases, and use of medications, including topical iodine, in premature infants ${ }^{13)}$. Therefore, it is highly recommended that hospitals perform repeat thyroid function tests (TFTs) in extremely premature infants ${ }^{14-17)}$. In practice, clinicians tend to conduct follow-ups regularly according to these guidelines for extremely early premature infants but not for late premature infants.

In relation to this institution's neonatal intensive care unit (NICU), the authors reported that patients had been exposed to iodine disinfectant before blood, cerebrospinal fluid, and urine culture tests; central line insertions; and periodic dressings. Given that there are many late preterm neonates, it is important to identify their individual physiology and formulate specific followup guidelines for improved outcomes. Due to a heightened awareness of this existing issue, medical staff aims to prevent iodine overload by implementing the institution's infection control guidelines enacted in 2015 , which substituted the standard iodine disinfectant with $0.5 \%$ to $2 \%$ chlorhexidine gluconate.

The primary purpose of this preliminary study was to compare the prevalence of thyroid dysfunction during the use and discon- tinuation of iodine disinfectants to determine its effects on the thyroid function of late preterm neonates. Furthermore, we analyzed specific risk factors to establish the basis of patient follow-up guidelines, with heightened consideration of thyroid hormone effects on neurodevelopment.

\section{MATERIALS AND METHODS}

\section{Design}

This single-center retrospective study was conducted to determine the clinical characteristics and risk factors of delayed thyroid dysfunction in late preterm infants. This study included late preterm infants admitted to a children's hospital with a tertiary referral neonatal center. The team specifically analyzed clinical features and risk factors of thyroid dysfunction by dividing infants into normal and abnormal groups based on TFT results performed at 2 to 4 weeks of age. Delayed thyrotropin level ele vation was defined as normal thyrotropin level at initial NTS, followed by elevation detected in a subsequent specimen ${ }^{18)}$.

\section{Study participants}

Participants were identified as late preterm infants with a gestational age (GA) of 34 to 36.6 weeks who underwent routine NTS and $\geq 2$ TFTs and were hospitalized in the NICU from January 2010 to June 2018. Infants with a maternal history of thyroid disease, major congenital anomalies, genetic disorders, metabolic disease, perinatal asphyxia, and congenital infection were excluded.

\section{NTS}

NTS was performed on the third day after birth using thyroidstimulating hormone (TSH). Capillary blood spots were soaked in filter paper designed for neonatal screening and dried at room temperature. These were examined with fluoroimmunoassay using VICTOR2 ${ }^{\mathrm{TM}}$ D or AutoDELFIA (PerkinElmer, Wallac Oy, Finland). According to the laboratory criteria, TSH levels $\geq 10$ $\mu \mathrm{IU} / \mathrm{mL}$ were classified as abnormal and reevaluated by TFTs.

\section{TFTs}

All premature infants who were admitted to the NICU underwent at least two TFTs, including measurement of TSH and free thyroxine (FT4) levels. TFT was first performed 1 week after birth and was again performed in the 2nd to 4th week regardless of 
previous results. Serum FT4 and TSH levels were measured by chemiluminescent microparticle immunoassay using Architect i2000SR (Abbott Laboratories, Abbott Park, IL, USA). The reference FT4 level was 0.7 to $1.48 \mathrm{ng} / \mathrm{dL}$. When the first TFT result showed FT4 levels $<0.7 \mathrm{ng} / \mathrm{dL}$ and TSH levels $>20 \mu \mathrm{U} / \mathrm{mL}$, hypothyroidism was confirmed and levothyroxine (LT4) therapy was initiated at a dose of 10 to $15 \mu \mathrm{g} / \mathrm{kg}$. Cutoff values in the second TFT performed at 2 to 4 weeks after birth were as follows: followup termination for normal FT4 levels and TSH level $<5 \mu \mathrm{U} / \mathrm{mL}$; follow-up after 2 weeks for normal FT4 levels and TSH level of 5 to $10 \mu \mathrm{U} / \mathrm{mL}$; and TSH levels $\geq 10 \mu \mathrm{U} / \mathrm{mL}$ were referred to endocrinologists and LT4 treatment was initiated.

\section{Data collection and outcome measurement}

All relevant demographic data of mothers and newborn infants were extracted from electronic medical records. Data collected from each patient included relevant information related to their individual anthropometric characteristics and any various complications that might pose a risk to the newborn. Maternal characteristics that were considered for this study included antenatal steroid use and the presence of gestational diabetes mellitus and pregnancy-induced hypertension.

To determine whether preterm infant hospitalization into the NICU was necessary and warranted, we considered the following criteria: GA $<35$ weeks, birth weight $<2 \mathrm{~kg}$, and the presence of certain acute problems such as respiratory distress, hypoglycemia, infection, and potential feeding disorders. While there were no changes in treatment guidelines for late premature infants in this study, there was a change in disinfectant type and use.

Small for gestational age (SGA) was defined as birth weight lower than the 10th percentile for sex and $\mathrm{GA}^{19)}$. The first echocardiography was performed 3 to 7 days after birth to confirm the presence of patent ductus arteriosus (PDA). Intraventricular hemorrhage was defined according to the classification by Papile et al. ${ }^{20)}$. Bronchopulmonary dysplasia was diagnosed when oxy. gen was required for $>4$ weeks after birth ${ }^{21)}$.

Time of diagnosis and TFTs in the thyroid dysfunction group was examined and divided into two distinctive time periods: iodine disinfectant use (period 1, January 2010 to February 2015) and iodine disinfectant discontinuation (period 2, March 2015 to June 2018). These individual periods were used to assess iodine use in the analysis of risk factors for thyroid dysfunction.

\section{Statistical analysis}

Statistical analyses were performed using SPSS version 21.0 (IBM Co., Armonk, NY, USA). Continuous variables were presented as mean and standard deviation (range), and categorical variables were presented as percentages. The thyroid dysfunction group was compared with the normal thyroid group using the independent Student's $t$-test for continuous variables and the chisquare test for categorical variables. Univariate logistic regression analysis was performed to analyze thyroid dysfunction risk indicators. Stepwise (forward and backward) multivariate logistic regression analysis was used to calculate adjusted odds ratios (AORs), 95\% confidence intervals (CIs), and $P$-values to adjust for potential confounders. Factors with a $P<0.2$ in the univariate analysis were included in the multivariate analysis. A $P<0.05$ was considered statistically significant.

\section{RESULTS}

\section{Study participants}

We included 262 of 295 late preterm infants who underwent $\geq 2$ TFTs (Figure 1). The most frequent cause of hospitalization was respiratory distress (77.5\%), followed by GA <35 weeks (56.5\%)

Table 1. Overall Morbidity of Late Preterm Infants on Admission

\begin{tabular}{|c|c|c|c|c|}
\hline Diagnosis & Total $(n=262)$ & Normal thyroid function $(n=242)$ & Thyroid dysfunction $(n=20)$ & $P$-value \\
\hline Gestational age $<35$ weeks & $148(56.5)$ & $136(56.2)$ & $12(60.0)$ & 0.742 \\
\hline Low birth weight $(<2,000 \mathrm{~g})$ & $82(31.3)$ & $72(29.8)$ & $10(50.0)$ & 0.061 \\
\hline Respiratory distress & $203(77.5)$ & $189(78.1)$ & $14(70.0)$ & 0.405 \\
\hline Feeding difficulty & $18(6.9)$ & $16(6.6)$ & $2(10.0)$ & 0.565 \\
\hline Hypoglycemia & $6(2.3)$ & $5(2.1)$ & $1(5.0)$ & 0.399 \\
\hline Temperature instability & $6(2.3)$ & $6(2.3)$ & 0 & 0.476 \\
\hline Fever & $3(1.1)$ & $3(1.2)$ & 0 & 0.617 \\
\hline
\end{tabular}

Values are expressed as number (\%). 
and low birth weight $(<2,000 \mathrm{~g})(31.3 \%)$. There were no significant differences in the causes of hospitalization between groups (Table 1).

The mean GA and birth weight of participants was $34.8 \pm 0.7$ weeks and 2,170 $\pm 454 \mathrm{~g}$, respectively. The normal thyroid function group consisted of 242 infants (92.4\%), and the thyroid dysfunc tion group comprised 20 infants (7.6\%); all infants with thyroid dysfunction had hyperthyrotropinemia. The thyroid dysfunction group had significantly lower birth weight $(1,870 \pm 472 \mathrm{~g}$ vs. $2,190 \pm 445 \mathrm{~g}, P=0.002)$, higher proportion of SGA infants $(45.0 \%$ vs. $18.2 \%, P=0.004)$, and abnormal NTS result (30.0\% vs. $5.8 \%, P=$ $0.001)$; no differences were observed for other clinical factors (Table 2).

\section{Thyroid dysfunction}

Twenty infants showed hyperthyrotropinemia at 2 to 4 weeks after birth. Delayed thyrotropin level elevation occurred in 14/242 patients (5.8\%) with normal TSH level on the 3rd day of life, 13/ 249 patients $(5.2 \%)$ with normal TSH level on the 7 th day after birth, and 10/234 patients (4.3\%) with normal TSH level on the 3rd and 7th day after birth (Figure 1).

The mean TSH level in the NTS was $4.74 \pm 3.95 \mu \mathrm{U} / \mathrm{mL}$ (range, 0.37 to 32.7$)$. In the 1 st week after birth, TSH level was $4.68 \pm 2.78$ $\mu \mathrm{U} / \mathrm{mL}$ (range, 0.28 to 19.82 ), and FT4 level was $1.65 \pm 0.37 \mathrm{ng} / \mathrm{dL}$ (range, 0.56 to 3.46 ). The mean time to thyroid dysfunction diagnosis was $24.3 \pm 14.6$ days (range, 12 to 69 ) after birth. Upon diagnosis, TSH level was $20.64 \pm 13.46 \mu \mathrm{U} / \mathrm{mL}$ (range, 10.14 to 71.80), and FT4 level was $1.13 \pm 0.25 \mathrm{ng} / \mathrm{dL}$ (range, 0.70 to 1.58 ).

Table 2. Comparison of Clinical Characteristics between the Normal and Thyroid Dysfunction Groups

\begin{tabular}{|c|c|c|c|c|}
\hline Characteristic & Total $(n=262)$ & Normal thyroid function $(n=242)$ & Thyroid dysfunction $(n=20)$ & $P$-value \\
\hline Gestational age (wk) & $34.8 \pm 0.7$ & $34.8 \pm 0.7$ & $34.9 \pm 0.8$ & 0.505 \\
\hline Birth weight (g) & $2,170 \pm 454$ & $2,190 \pm 445$ & $1,870 \pm 472$ & 0.002 \\
\hline Male sex & $134(51.1)$ & $127(52.5)$ & $7(35.0)$ & 0.133 \\
\hline Multiple gestation & $73(27.9)$ & $67(27.7)$ & $6(30.0)$ & 0.824 \\
\hline SGA & $53(20.2)$ & $44(18.2)$ & $9(45.0)$ & 0.004 \\
\hline Caesarean section & $191(72.9)$ & $176(72.7)$ & $15(75.0)$ & 0.826 \\
\hline \multicolumn{5}{|l|}{ Maternal characteristic } \\
\hline Antenatal steroids & $1(0.4)$ & $1(0.4)$ & 0 & 0.773 \\
\hline GDM & $24(9.2)$ & $24(9.9)$ & 0 & 0.139 \\
\hline $\mathrm{PIH}$ & $54(20.6)$ & $53(21.9)$ & $1(5.0)$ & 0.073 \\
\hline 1 min Apgar score & $5.9 \pm 1.5$ & $5.9 \pm 1.5$ & $5.9 \pm 1.4$ & 0.990 \\
\hline 5 min Apgar score & $7.7 \pm 1.1$ & $7.7 \pm 1.1$ & $7.7 \pm 1.3$ & 0.901 \\
\hline RDS & $60(22.9)$ & $54(22.3)$ & $6(30.0)$ & 0.432 \\
\hline PDA & $18(6.9)$ & $15(6.2)$ & $3(15.0)$ & 0.135 \\
\hline IVH ( $\geq$ grade III) & $3(1.1)$ & $3(1.2)$ & 0 & 0.163 \\
\hline BPD & $8(3.1)$ & $7(2.9)$ & $1(5.0)$ & 0.599 \\
\hline Culture proven sepsis & $1(0.4)$ & $1(0.4)$ & 0 & 0.920 \\
\hline Oxygen supply & $202(77.1)$ & $187(77.3)$ & $15(75.0)$ & 0.816 \\
\hline Oxygen supply (d) & $5.6 \pm 6.9$ & $5.6 \pm 6.9$ & $5.2 \pm 7.0$ & 0.764 \\
\hline Mechanical ventilation & $101(38.5)$ & $93(38.4)$ & $8(40.0)$ & 0.890 \\
\hline Mechanical ventilation (d) & $1.4 \pm 3.0$ & $1.4 \pm 3.0$ & $1.0 \pm 2.2$ & 0.532 \\
\hline Dopamine & $14(5.3)$ & $13(5.4)$ & $1(5.0)$ & 0.943 \\
\hline Dopamine (d) & $0.2 \pm 1.4$ & $0.3 \pm 1.4$ & $0.1 \pm 0.5$ & 0.641 \\
\hline Central line & $137(52.3)$ & $126(52.1)$ & $11(55.0)$ & 0.801 \\
\hline Central line (d) & $7.7 \pm 10.4$ & $7.1 \pm 9.1$ & $14.7 \pm 19.9$ & 0.106 \\
\hline Hospital stay (d) & $19.9 \pm 13.7$ & $19.4 \pm 13.0$ & $26.2 \pm 19.2$ & 0.136 \\
\hline Abnormal NTS & $20(7.6)$ & $14(5.8)$ & $6(30.0)$ & 0.001 \\
\hline
\end{tabular}

Values are expressed as mean \pm standard deviation or number $(\%)$.

Abbreviations: SGA, small for gestational age; GDM, gestational diabetes mellitus; PIH, pregnancy-induced hypertension; RDS, respiratory distress syndrome; PDA, patent ductus arteriosus; IVH, intraventricular hemorrhage; BPD, bronchopulmonary dysplasia; NTS, neonatal thyroid screening. 
A total of 44 preterm infants with TSH levels of 5 to $10 \mu \mathrm{U} / \mathrm{mL}$ in the 2 nd week after birth underwent a third TFT, and 40 preterm infants had TSH levels of $3.37 \pm 1.22 \mu \mathrm{U} / \mathrm{mL}$ (range, 0.87 to 5.81 ) and FT4 levels of $1.25 \pm 0.25 \mathrm{ng} / \mathrm{dL}$ (range, 0.9 to 2.1 ) at $41.1 \pm 10.8$ days (range, 23 to 61) after birth; the follow-up was terminated. In contrast, four preterm infants received treatment with TSH levels of $17.62 \pm 10.45 \mu \mathrm{U} / \mathrm{mL}$ (range, 10.14 to 32.39 ) and FT4 levels of $1.25 \pm 0.21 \mathrm{ng} / \mathrm{dL}$ (range, 0.94 to 1.42 ) at $49.3 \pm 14.2$ days (range, 38 to 69) after birth.

Period 1 showed a higher incidence of thyroid dysfunction (12.6\% vs. $2.4 \%, P=0.002$ ) and delayed thyrotropin level elevation ( $8.9 \%$ vs. $1.6 \%, P=0.009)$, lower mean birth weight $(2,110 \pm 440 \mathrm{~g}$ vs. $2,230 \pm 462 \mathrm{~g}, P=0.029$ ), and more male patients (57.8\% vs. $44.1 \%$, $P=0.027)$. There were no clinically significant differences in other factors (Table 3 ).

\section{Risk factors}

The variables used in the multiple logistic regression analysis were as follows: low birth weight $(<2,000 \mathrm{~g})$, male sex, SGA, presence of PDA, period 1, and abnormal NTS result, as these factors

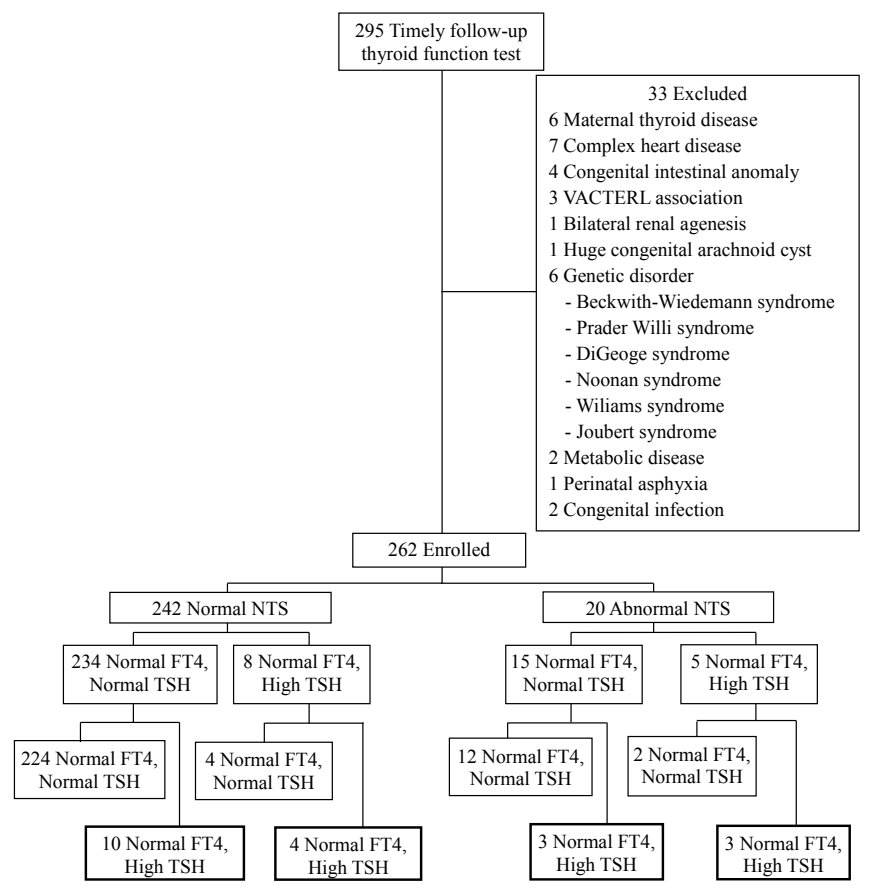

Figure 1. Flowchart of study population selection. Abbreviations: VACTERL association, associated vertebral defects, anal atresia, cardiac defects, tracheoesophageal fistula, renal anomalies, and limb abnormalities; NTS, neonatal thyroid screening; FT4, free thyroxine; TSH, thyroid-stimulating hormone (thyrotropin). had $P$-values $<0.2$ in the univariate analysis (Table 4 ). SGA, period 1 , and abnormal NTS result were significant risk factors in the multivariate analysis ([AOR, $4.27 ; 95 \% \mathrm{CI}, 1.45$ to $12.54 ; P=0.008]$, [AOR, 8.24; 95\% CI, 2.05 to 33.09; $P=0.003$ ], and [AOR, 7.80; 95\% CI, 2.17 to 28.08; $P=0.002$ ], respectively) (Table 5).

Table 3. Comparison of Clinical Characteristics between Periods

\begin{tabular}{|c|c|c|c|}
\hline Characteristic & $\begin{array}{c}\text { Period 1* } \\
(n=135)\end{array}$ & $\begin{array}{c}{\text { Period } 2^{\dagger}}_{(n=127)}\end{array}$ & $\begin{array}{c}P \text { - } \\
\text { value }\end{array}$ \\
\hline Thyroid dysfunction & $17(12.6)$ & $3(2.4)$ & 0.002 \\
\hline Delayed thyrotropin elevation & $12(8.9)$ & $2(1.6)$ & 0.009 \\
\hline Gestational age (wk) & $34.8 \pm 0.7$ & $34.7 \pm 0.7$ & 0.097 \\
\hline Birth weight (g) & $2,110 \pm 440$ & $2,230 \pm 462$ & 0.029 \\
\hline Male sex & $78(57.8)$ & $56(44.1)$ & 0.027 \\
\hline Multiple gestation & $43(31.9)$ & $30(23.6)$ & 0.138 \\
\hline SGA & $33(24.4)$ & $21(16.5)$ & 0.114 \\
\hline Caesarean section & $94(69.6)$ & $97(76.4)$ & 0.219 \\
\hline \multicolumn{4}{|l|}{ Maternal characteristic } \\
\hline Antenatal steroids & 0 & $1(0.8)$ & 0.302 \\
\hline GDM & $10(7.4)$ & $14(11.0)$ & 0.311 \\
\hline $\mathrm{PIH}$ & $24(17.8)$ & $30(23.6)$ & 0.243 \\
\hline 1 min Apgar score & $6.1 \pm 1.5$ & $5.7 \pm 1.5$ & 0.062 \\
\hline 5 min Apgar score & $7.7 \pm 1.1$ & $7.6 \pm 1.2$ & 0.180 \\
\hline RDS & $27(20.0)$ & $33(26.0)$ & 0.249 \\
\hline PDA & $10(7.4)$ & $8(6.3)$ & 0.723 \\
\hline IVH ( $\geq$ grade III) & $1(0.7)$ & $2(1.6)$ & 0.017 \\
\hline BPD & $4(3.0)$ & $4(3.1)$ & 0.930 \\
\hline Culture proven sepsis & 0 & $1(0.8)$ & 0.343 \\
\hline Oxygen supply & $98(72.6)$ & $104(81.9)$ & 0.073 \\
\hline Oxygen supply (d) & $5.4 \pm 6.4$ & $5.9 \pm 7.3$ & 0.550 \\
\hline Mechanical ventilation & $46(34.1)$ & $55(43.3)$ & 0.125 \\
\hline Mechanical ventilation (d) & $0.9 \pm 1.5$ & $1.9 \pm 4.0$ & 0.010 \\
\hline Dopamine & $7(5.2)$ & $7(5.5)$ & 0.906 \\
\hline Dopamine (d) & $0.2 \pm 0.9$ & $0.3 \pm 1.7$ & 0.529 \\
\hline Central line & $70(51.9)$ & $67(52.8)$ & 0.884 \\
\hline Central line (d) & $7.9 \pm 11.2$ & $7.5 \pm 9.6$ & 0.793 \\
\hline Hospital stay (d) & $20.6 \pm 13.3$ & $19.1 \pm 14.1$ & 0.359 \\
\hline Abnormal NTS & $11(8.1)$ & $9(7.1)$ & 0.746 \\
\hline
\end{tabular}

Values are expressed as number (\%) or mean \pm standard deviation.

*Period 1, iodinated disinfectant use period (from January 2010 to February 2015); ${ }^{\dagger}$ Period 2, iodinated disinfectant discontinuation period (from March 2015 to June 2018).

Abbreviations: SGA, small for gestational age; GDM, gestational diabetes mellitus; PIH, pregnancy-induced hypertension; RDS, respiratory distress syndrome; PDA, patent ductus arteriosus; IVH, intraventricular hemorrhage; BPD, bronchopulmonary dysplasia; NTS, neonatal thyroid screening. 
Table 4. Univariate Logistic Regression Analysis of the Factors Associated with Delayed Thyrotropin Level Elevation in Late Preterm Infants

\begin{tabular}{lccc}
\hline Variable & Odds ratio & $95 \%$ CI & P-value \\
\hline Gestational age (wk) & & & \\
$\quad<35$ & 1.13 & $0.45-2.87$ & 0.796 \\
$\geq 35$ & Reference & Reference & Reference \\
Birth weight (g) & & & \\
$<2,000$ & 2.27 & $0.91-5.69$ & 0.080 \\
$\geq 2,000$ & Reference & Reference & Reference \\
Male sex & 0.49 & $0.19-1.26$ & 0.140 \\
Multiple gestation & 1.12 & $0.41-3.03$ & 0.825 \\
Caesarean section & 1.13 & $0.39-3.21$ & 0.826 \\
SGA & 4.50 & $1.76-11.46$ & 0.002 \\
1 min Apgar score & & & \\
0-6 & 0.99 & $0.39-2.51$ & 0.982 \\
7-10 & Reference & Reference & Reference \\
5 min Apgar score & & & \\
0-6 & 0.84 & $0.18-3.80$ & 0.818 \\
7-10 & Reference & Reference & Reference \\
RDS & 1.49 & $0.55-4.07$ & 0.434 \\
PDA & 2.67 & $0.70-10.14$ & 0.149 \\
Period of iodine disinfectant use & 5.95 & $1.70-20.84$ & 0.005 \\
Abnormal NTS & 6.98 & $2.32-20.92$ & 0.001 \\
\hline Abbrevation & & & \\
\hline
\end{tabular}

Abbreviations: CI, confidence interval; SGA, small for gestational age; RDS, respiratory distress syndrome; PDA, patent ductus arteriosus; NTS, neonatal thyroid screening.

Table 5. Risk Factors Associated with Delayed Thyrotropin Level Elevation in Late Preterm Infants

\begin{tabular}{lccc}
\hline Variable & $\begin{array}{c}\text { Adjusted } \\
\text { odds ratio }\end{array}$ & $95 \%$ CI & $P$-value \\
\hline SGA & 4.27 & $1.45-12.54$ & 0.008 \\
Period of iodine disinfectant use & 8.24 & $2.05-33.09$ & 0.003 \\
Abnormal NTS & 7.80 & $2.17-28.08$ & 0.002
\end{tabular}

Low birth weight $(<2,000 \mathrm{~g})$, male, SGA, patent ductus arteriosus, period of iodine disinfectant use and abnormal NTS were included in the multivariate logistic regression analysis.

Abbreviations: CI, confidence interval; SGA, small for gestational age; NTS, neonatal thyroid screening.

\section{DISCUSSION}

Thyroid dysfunction was noted in $7.6 \%$ of late premature infants 2 weeks after birth, and all were hyperthyrotropinemia. Additionally, delayed thyrotropin level elevation occurred in $5.8 \%$ of patients who showed normal NTS result. The incidence of thyroid dysfunction during iodine administration was $12.6 \%$, while that during iodine discontinuation was $2.4 \%$. This significant discrepancy indicates that the use of iodine disinfectant is a risk factor for delayed hyperthyrotropinemia in late preterm infants.

Very low birth weight (VLBW) infants had 14 times higher incidence of transient hypothyroidism than infants weighing $>1,500$ $\mathrm{g}$ at birth, and most showed delayed TSH level elevation ${ }^{22-24)}$. In a 2015 study, 9.8\% (24/246) of VLBW infants had elevated TSH level, and $91.6 \%$ of these $(22 / 24)$ had delayed TSH level elevation ${ }^{25)}$. Kaluarachchi et al. ${ }^{26)}$ also evaluated infants with GA $<30$ weeks and reported that $6.9 \%$ had congenital hypothyroidism with delayed TSH level elevation. While it was difficult to compare the given values due to varying TFT times and cutoff levels, the occurrence of delayed TSH level elevation in late premature infants during iodine administration was similar to those in previous studies including younger infants. This suggests a need for secondary TFTs for late preterm infants with a history of iodine disinfectant use.

Excessive iodine can cause thyrotropin level elevation ${ }^{27)}$. Normally, when there is excessive iodine, thyroid iodine uptake and iodination of tyrosine cease production to prevent overproduction of thyroid hormone. This defense mechanism, known as the Wolff-Chaikoff effect, does not mature until 36 to 40 weeks' gestation. Furthermore, premature infants are exceedingly vulnerable to iodine exposure due to increased iodine absorption through permeable skin and delayed iodine excretion through the kidneys ${ }^{28,29)}$. Results from this study showed that 17 of 20 patients ( 85 \%) with hyperthyrotropinemia were hospitalized during iodine disinfectant use. Additionally, Aitken and Williams ${ }^{28)}$ found that the incidence of hypothyroidism and hyperthyrotropinemia was significantly higher in premature infants with GA $<32$ weeks ( $12 \%$ to $33 \%$ ) and who were exposed to iodine disinfectants. Our results were similar to those of previous studies on extremely premature infants and showed that care should be taken when using iodine disinfectants in late preterm infants. If they must be used, followup TFT is highly recommended.

In this study, we analyzed clinical differences in patients depending on the period, as there was a higher proportion of male and SGA infants in period 1, but there were no differences in diseases or length of hospital stay. We also evaluated the number of patients who had central lines and the duration; this is because the frequency of disinfectant use may increase with central line insertion and maintenance ${ }^{16)}$. However, we found no difference 
between the two periods. This evidence suggests that ordinary use of iodine disinfectants for culture testing or dressings can affect thyroid function of late preterm infants. Other sources of iodine include not only iodine disinfectants used on mothers before delivery but also breast milk. For example, seaweed soup is traditionally consumed by Korean mothers post-delivery and is rich in iodine; therefore, we should also consider the effect of breastfeeding ${ }^{30)}$. However, for the purpose of this study, this claim is unsubstantiated because urinary iodine concentration tests were never performed.

SGA was also a significant risk factor of thyroid dysfunction. Bagnoli et al. ${ }^{31)}$ analyzed NTS performed on 14,092 (759 SGA, 5.38 \%) newborns and reported significantly lower thyroxine (T4) level in preterm and term SGA infants. They also noted significantly higher TSH levels in term and preterm SGA infants. In both preterm and term SGA infants, NTS recall was often needed, but hypothyroxinemia in SGA infants was mostly transient. Their insight led them to believe that malnutrition during specific fetal periods causes delays in thyroid gland development and low T4 level in the early neonatal period. However, because TSH level is increased by the pituitary gland, it is considered less affected by nutrition when responding to lower T4 level. In addition to this observation, it was reported that adequate nutrition after birth naturally normalizes TFT results in the following months ${ }^{31,32)}$. Uchiyama et al. ${ }^{33)}$ conducted a study that included premature infants, aged 23 to 36.6 weeks, with a median/mean GA of 27.4/ 28.6 weeks and concluded that SGA was a risk factor for delayed TSH level elevation in newborns weighing $<2 \mathrm{~kg}$ at birth.

Given the results of our study with GA of 34 to 36 weeks, SGA may be a risk factor for delayed TSH level elevation in premature infants of any GA. This is the first study that shows SGA is a risk factor of thyroid dysfunction in late preterm infants. There are limited studies on SGA TFTs; therefore, further investigations into the thyroid hormone profile for SGA and criteria, efficacy, and safety of LT4 treatment are needed.

Clinically abnormal NTS results are broadly utilized to assist tracking. For example, 14/20 patients with confirmed thyroid dysfunction had normal NTS results but showed abnormalities 2 weeks after birth. On average, $27.5 \pm 17.1$ days (range, 12 to 69 ) after birth, 14 patients with previously normal NTS results showed TSH level of $18.57 \pm 6.58 \mu \mathrm{U} / \mathrm{mL}$ (range, 10.14 to 32.39 ), which is relatively high when compared to the normal TSH level of late preterm infants 2 weeks after birth ${ }^{34)}$. Our results show that TSH level, which should decrease 2 weeks after birth, has a delayed elevation in late preterm infants and suggests a need for repeated tests. Several studies on VLBW infants showed delayed thyroid dysfunction approximately 3 weeks after birth ${ }^{22,23)}$. Opinions vary regarding the recommended rescreening time for TFT, and these variances can range between 2 and 6 weeks after birth $^{14,35-37)}$. Hashemipour et al. ${ }^{37}$ reviewed documentation on the screening process of preterm congenital hypothyroidism and suggested that repeated screening is necessary. A TSH level of 10 $\mathrm{mU} / \mathrm{L}$ was defined as the positive cutoff level; a TSH level of 10 to $15 \mathrm{mU} / \mathrm{L}$ after one normal TSH level might be indicative of delayed TSH level elevation that increases the possibility of $\mathrm{CH}$. Further research is needed to explore whether 2 to 4 weeks after birth, regardless of GA, is the most critical point in thyroid func tion. For late preterm infants with a history of NICU hospitalization, TFTs at 2 to 4 weeks must be considered even when the first NTS result is normal.

Our study identified that delayed thyroid dysfunction in all patients was hyperthyrotropinemia. As TSH is the most sensitive indicator of thyroid function, LT4 treatments are recommended in case of consistently elevated TSH levels. One study reported that neonatal hyperthyrotropinemia was a risk factor for persis tent hyperthyrotropinemia accompanied with developmental delay ${ }^{10)}$ Hyperthyrotropinemia observed in premature infants was also related to long-term developmental delays, while other studies reported that early termination of treatment was possible in most infants ${ }^{38-40)}$. Due to inconsistencies with researcher recommendations and vagueness of specific guidelines, it would be advantageous to execute large-scale longitudinal studies that include long-term developmental follow-up.

The authors of this study recognize that there were significant limitations. First, this was a single-center study with a limited number of participants. Second, due to the retrospective nature of the study, we were unable to validate the exact iodine level in the body. Third, this study was conducted considering iodine exposure as a factor affecting thyroid function but did not include long-term developmental follow-up. Finally, many late preterm infants with acute problems necessitating NICU hospitalization were included, as we cannot eliminate the impact of illness on thyroid function. However, this study is significant in that it provides a basis for the necessity of secondary thyroid screening by illustrating the incidence of delayed thyrotropin level elevation in late preterm infants, as there are few studies on TFT of late preterm infants.

Therefore, our retrospective study reported that late preterm 
infants who were SGA or exposed to iodine disinfectants showed delayed thyrotropin level elevation with high incidence and identified the need for secondary TFT 2 to 4 weeks after birth. It is suggested that well-designed, large-scale studies are necessary to establish TFT guidelines for late preterm infants. We also con cluded that prospective research focused on the relationship between long-term neurodevelopment outcomes and TFT changes associated with iodine exposure and iodine levels in the body is needed.

\section{ARTICLE INFORMATION}

\section{Ethical statement}

This study was approved by the Institutional Review Board of Pusan National University Yangsan Hospital, and the requirement for informed consent was waived (IRB No. 05-2018-119).

\section{Conflicts of interest}

No potential conflict of interest relevant to this article was reported.

\section{Author contributions}

Conception or design: Y.M.H.

Acquisition, analysis, or interpretation of data: M.H.J., N.L., M.H.B., K.H.P., S.Y.B.

Drafting the work or revising: Y.M.H., M.H.J.

Final approval of the manuscript: M.H.J., N.L., M.H.B., Y.M.H., K.H.P., S.Y.B.

\section{ORCID}

Mun Hui Jeong https://orcid.org/0000-0002-5073-7276

Young Mi Han https://orcid.org/0000-0002-6120-0490

\section{Acknowledgments}

None

\section{REFERENCES}

1. Blencowe H, Cousens S, Oestergaard MZ, Chou D, Moller AB, Narwal R, et al. National, regional, and worldwide estimates of preterm birth rates in the year 2010 with time trends since 1990 for selected countries: a systematic analysis and implications.
Lancet 2012;379:2162-72.

2. Woythaler M. Neurodevelopmental outcomes of the late preterm infant. Semin Fetal Neonatal Med 2019;24:54-9.

3. Raju TN, Higgins RD, Stark AR, Leveno KJ. Optimizing care and outcome for late-preterm (near-term) infants: a summary of the workshop sponsored by the National Institute of Child Health and Human Development. Pediatrics 2006;118:1207-14.

4. Kugelman A, Colin AA. Late preterm infants: near term but still in a critical developmental time period. Pediatrics 2013;132: 741-51.

5. McGowan JE, Alderdice FA, Holmes VA, Johnston L. Early childhood development of late-preterm infants: a systematic review. Pediatrics 2011;127:1111-24.

6. Heinonen K, Eriksson JG, Lahti J, Kajantie E, Pesonen AK, Tuovinen S, et al. Late preterm birth and neurocognitive performance in late adulthood: a birth cohort study. Pediatrics 2015; 135:e818-25.

7. Shah P, Kaciroti N, Richards B, Oh W, Lumeng JC. Developmental outcomes of late preterm infants from infancy to kindergarten. Pediatrics 2016;138:e20153496.

8. Celik IH, Demirel G, Canpolat FE, Dilmen U. A common problem for neonatal intensive care units: late preterm infants, a prospective study with term controls in a large perinatal center. J Matern Fetal Neonatal Med 2013;26:459-62.

9. Fleming PF, Arora P, Mitting R, Aladangady N. A national survey of admission practices for late preterm infants in England. BMC Pediatr 2014;14:150.

10. Cuestas E, Gaido MI, Capra RH. Transient neonatal hyperthyrotropinemia is a risk factor for developing persistent hyperthyrotropinemia in childhood with repercussion on developmental status. Eur J Endocrinol 2015;172:483-90.

11. Oppenheimer JH, Schwartz HL. Molecular basis of thyroid hormone-dependent brain development. Endocr Rev 1997;18: 462-75.

12. Grosse SD, Van Vliet G. Prevention of intellectual disability through screening for congenital hypothyroidism: how much and at what level? Arch Dis Child 2011;96:374-9.

13. Van Wassenaer AG, Kok JH. Hypothyroxinaemia and thyroid function after preterm birth. Semin Neonatol 2004;9:3-11.

14. American Academy of Pediatrics, Rose SR; Section on Endocrinology and Committee on Genetics, American Thyroid Association, Brown RS; Public Health Committee, Lawson Wilkins Pediatric Endocrine Society, Foley T, et al. Update of newborn screening and therapy for congenital hypothyroidism. Pediatrics 2006;117:2290-303.

15. Mass Screening Committee; Japanese Society for Pediatric Endocrinology; Japanese Society for Mass Screening, Nagasaki K, Minamitani K, Anzo M, et al. Guidelines for mass screening of congenital hypothyroidism (2014 revision). Clin Pediatr 
Endocrinol 2015;24:107-33.

16. Kucharska AM, Ben-Skowronek I, Walczak M, Oltarzewski M, Szalecki M, Jackowska T, et al. Congenital hypothyroidism: Polish recommendations for therapy, treatment monitoring, and screening tests in special categories of neonates with increased risk of hypothyroidism. Endokrynol Pol 2016;67:53647.

17. Leger J, Olivieri A, Donaldson M, Torresani T, Krude H, van Vliet G, et al. European Society for Paediatric Endocrinology consensus guidelines on screening, diagnosis, and management of congenital hypothyroidism. Horm Res Paediatr 2014;81:80-103.

18. Zung A, Yehieli A, Blau A, Almashanu S. Characteristics of delayed thyroid stimulating hormone elevation in neonatal intensive care unit newborns. J Pediatr 2016;178:135-40.

19. Fenton TR, Kim JH. A systematic review and meta-analysis to revise the Fenton growth chart for preterm infants. BMC Pediatr 2013;13:59.

20. Papile LA, Burstein J, Burstein R, Koffler H. Incidence and evolution of subependymal and intraventricular hemorrhage: a study of infants with birth weights less than 1,500 gm. J Pediatr 1978;92:529-34.

21. Kinsella JP, Greenough A, Abman SH. Bronchopulmonary dysplasia. Lancet 2006;367:1421-31.

22. Larson C, Hermos R, Delaney A, Daley D, Mitchell M. Risk factors associated with delayed thyrotropin elevations in congenital hypothyroidism. J Pediatr 2003;143:587-91.

23. Woo HC, Lizarda A, Tucker R, Mitchell ML, Vohr B, Oh W, et al. Congenital hypothyroidism with a delayed thyroid-stimulating hormone elevation in very premature infants: incidence and growth and developmental outcomes. J Pediatr 2011;158:53842.

24. Sun X, Lemyre B, Nan X, Harrold J, Perkins SL, Lawrence SE, et al. Free thyroxine and thyroid-stimulating hormone reference intervals in very low birth weight infants at 3-6 weeks of life with the Beckman Coulter Unicel DxI 800. Clin Biochem 2014;47:168.

25. Lee JH, Kim SW, Jeon GW, Sin JB. Thyroid dysfunction in very low birth weight preterm infants. Korean J Pediatr 2015;58:2249.

26. Kaluarachchi DC, Colaizy TT, Pesce LM, Tansey M, Klein JM. Congenital hypothyroidism with delayed thyroid-stimulating hormone elevation in premature infants born at less than 30 weeks gestation. J Perinatol 2017;37:277-82.

27. Linder N, Davidovitch N, Reichman B, Kuint J, Lubin D, Meyerovitch J, et al. Topical iodine-containing antiseptics and sub clinical hypothyroidism in preterm infants. J Pediatr 1997;131: 434-9.

28. Aitken J, Williams FL. A systematic review of thyroid dysfunction in preterm neonates exposed to topical iodine. Arch Dis Child
Fetal Neonatal Ed 2014;99:F21-8.

29. Ares S, Quero J, de Escobar GM. Iodine balance, iatrogenic excess, and thyroid dysfunction in premature newborns. Semin Perinatol 2008;32:407-12.

30. Chung HR, Shin CH, Yang SW, Choi CW, Kim BI. Subclinical hypothyroidism in Korean preterm infants associated with high levels of iodine in breast milk. J Clin Endocrinol Metab 2009;94: 4444-7.

31. Bagnoli F, Farmeschi L, Peruzzi L, Musaraf A, Paffetti P, Badii S, et al. Altered thyroid function in small for gestational age newborns: study based on screening test for congenital hypothyroidism. J Pediatr Sci 2010;4:e55.

32. Franco B, Laura F, Sara N, Salvatore G. Thyroid function in small for gestational age newborns: a review. J Clin Res Pediatr Endocrinol 2013;5 Suppl 1:2-7.

33. Uchiyama A, Watanabe H, Nakanishi H, Totsu S. Small for gestational age is a risk factor for the development of delayed thyrotropin elevation in infants weighing less than 2000 g. Clin Endocrinol (Oxf) 2018;89:431-6.

34. Aktas ON, Gursoy T, Soysal E, Esencan E, Ercin S. Thyroid hormone levels in late preterm, early term and term infants: a study with healthy neonates revealing reference values and factors affecting thyroid hormones. J Pediatr Endocrinol Metab 2017;30:1191-6.

35. Vigone MC, Caiulo S, Di Frenna M, Ghirardello S, Corbetta C, Mosca F, et al. Evolution of thyroid function in preterm infants detected by screening for congenital hypothyroidism. J Pediatr 2014;164:1296-302.

36. Bijarnia S, Wilcken B, Wiley VC. Newborn screening for congenital hypothyroidism in very-low-birth-weight babies: the need for a second test. J Inherit Metab Dis 2011;34:827-33.

37. Hashemipour M, Hovsepian S, Ansari A, Keikha M, Khalighinejad P, Niknam N. Screening of congenital hypothyroidism in preterm, low birth weight and very low birth weight neonates: a systematic review. Pediatr Neonatol 2018;59:3-14.

38. Chung ML, Yoo HW, Kim KS, Lee BS, Pi SY, Lim G, et al. Thyroid dysfunctions of prematurity and their impacts on neurodevelopmental outcome. J Pediatr Endocrinol Metab 2013;26: 449-55.

39. Lim G, Lee YK, Han HS. Early discontinuation of thyroxine therapy is possible in most very low-birthweight infants with hypothyroidism detected by screening. Acta Paediatr 2014;103: e123-9.

40. Jung JM, Jin HY, Chung ML. Feasibility of an early discontinuation of thyroid hormone treatment in very-low-birthweight infants at risk for transient or permanent congenital hypothyroidism. Horm Res Paediatr 2016;85:131-9. 